\title{
The largest singletons in weighted set partitions and its applications
}

\author{
Yidong Sun $\|^{\dagger}$ and Yanjie Xu \\ Department of Mathematics, Dalian Maritime University, P.R. China \\ received $16^{\text {th }}$ October 2010, revised $26^{\text {th }}$ November 2011, accepted $29^{\text {th }}$ November 2011.
}

Recently, Deutsch and Elizalde studied the largest fixed points of permutations. Motivated by their work, we consider the analogous problems in weighted set partitions. Let $A_{n, k}(\mathbf{t})$ denote the total weight of partitions on $[n+1]=\{1,2, \ldots, n+1\}$ with the largest singleton $\{k+1\}$. In this paper, explicit formulas for $A_{n, k}(\mathbf{t})$ and many combinatorial identities involving $A_{n, k}(\mathbf{t})$ are obtained by umbral operators and combinatorial methods. In particular, the permutation case leads to an identity related to tree enumerations, namely,

$$
\sum_{k=0}^{n}\left(\begin{array}{l}
n \\
k
\end{array}\right) D_{k+1}(n+1)^{n-k}=n^{n+1}
$$

where $D_{k}$ is the number of permutations of $[k]$ with no fixed points.

Keywords: Set partition, Bell polynomial, Permutation, Derangement.

\section{Introduction}

A partition of a set $[n]=\{1,2, \ldots, n\}$ is a collection $\pi=\left\{\mathbb{B}_{1}, \mathbb{B}_{2}, \ldots, \mathbb{B}_{r}\right\}$ of nonempty and mutually disjoint subsets of $[n]$, called blocks, whose union is $[n]$. For a block $\mathbb{B}$, we denote by $|\mathbb{B}|$ the size of the block $\mathbb{B}$, that is the number of the elements in the block $\mathbb{B}$. A block $\mathbb{B}$ will be called singleton if $|\mathbb{B}|=1$. If $\{k\}$ is a singleton of a partition, we denote it by $k$ for short. If $|\mathbb{B}|=j$, we assign a weight $t_{j}$ for $\mathbb{B}$. The weight $w(\pi)$ of a partition $\pi$ is defined to be the product of the weight of each block of $\pi$.

It is well known that the weight of partitions of $[n]$ with $r$ blocks is the partial Bell polynomial $\mathcal{B}_{n, r}\left(t_{1}, t_{2}, \ldots\right)[3]$ on the variables $\left\{t_{j}\right\}_{j \geq 1}$, that is

$$
\mathcal{B}_{n, r}\left(t_{1}, t_{2}, \ldots\right)=\sum_{\kappa_{n}(r)} \frac{n !}{r_{1} ! r_{2} ! \cdots r_{n} !}\left(\frac{t_{1}}{1 !}\right)^{r_{1}}\left(\frac{t_{2}}{2 !}\right)^{r_{2}} \cdots\left(\frac{t_{n}}{n !}\right)^{r_{n}}
$$

\footnotetext{
${ }^{\dagger}$ Email: sydmatheyahoo.com.cn. This work is supported by The National Science Foundation of China (Grant No. 10801020), by the Fundamental Research Funds for the Central Universities (Grant No. 2011QN056), and by The National Science Foundation of Liaoning Province (Grant No. 201102015).
}

1365-8050 @ 2011 Discrete Mathematics and Theoretical Computer Science (DMTCS), Nancy, France 
where the summation $\kappa_{n}(r)$ is over all the nonnegative integer solutions of $r_{1}+r_{2}+\cdots+r_{n}=r$ and $r_{1}+2 r_{2}+\cdots+n r_{n}=n$. The total weight for partitions of $[n]$ is the complete Bell polynomial

$$
\mathcal{Y}_{n}(\mathbf{t})=\mathcal{Y}_{n}\left(t_{1}, t_{2}, \ldots\right)=\sum_{r=0}^{n} \mathcal{B}_{n, r}\left(t_{1}, t_{2}, \ldots\right)
$$

which has the exponential generating function

$$
\mathcal{Y}(\mathbf{t} ; x)=\sum_{n \geq 0} \mathcal{Y}_{n}\left(t_{1}, t_{2}, \ldots\right) \frac{x^{n}}{n !}=\exp \left(\sum_{j \geq 1} t_{j} \frac{x^{j}}{j !}\right) .
$$

Let $\mathbb{A}_{n, k}$ denote the set of partitions of $[n+1]$ with the largest singleton $k+1$. Let $A_{n, k}(\mathbf{t})$ denote the total weight of partitions in $\mathbb{A}_{n, k}$. Clearly,

$$
A_{n, 0}(\mathbf{t})=t_{1} \mathcal{Y}_{n}\left(0, t_{2}, \ldots\right) \text { and } A_{n, n}(\mathbf{t})=t_{1} \mathcal{Y}_{n}\left(t_{1}, t_{2}, \ldots\right),
$$

where $\mathcal{Y}_{n}\left(0, t_{2}, \ldots\right)$ is the weight of partitions of $[n]$ without singletons.

Recently, Deutsch and Elizalde [4] studied the largest fixed points of permutations, which is the special case when $t_{j}=(j-1)$ ! for $j \geq 1$. Later, Sun and $\mathrm{Wu}$ [17] considered the largest singletons in set partitions, which is the special case when $t_{j}=1$ for $j \geq 1$.

In this paper we will investigate the largest singletons in weighted set partitions generally. The next section is devoted to studying the properties of $A_{n, k}(\mathbf{t})$, involving its explicit formulas and many combinatorial identities for $A_{n, k}(\mathbf{t})$. In the third section, we consider the permutation case, i.e., the special case when $t_{j}=(j-1)$ ! for $j \geq 1$, and derive a surprising identity analogous to the Riordan identity related to tree enumerations.

\section{The properties of $A_{n, k}(\mathbf{t})$}

According to the definition of $A_{n, k}(\mathbf{t})$, for any weighted partition $\pi$ of $[n+1]$ with the largest singleton $k+1$, if $k$ is also a singleton, delete the singleton $k+1$ and subtracting one from all the entries larger than $k+1$, we obtain a partition of $[n]$ with the largest singleton $k$. This contributes the weight $t_{1} A_{n-1, k-1}(\mathbf{t})$; if $k$ is not a singleton, exchange $k$ and $k+1$, we obtain a partition of $[n+1]$ with the largest singleton $k$. This contributes the weight $A_{n, k-1}(\mathbf{t})$. Consequently, we obtain a recurrence for $n, k \geq 1$,

$$
A_{n, k}(\mathbf{t})=A_{n, k-1}(\mathbf{t})+t_{1} A_{n-1, k-1}(\mathbf{t})
$$

with the initial conditions $A_{n, 0}(\mathbf{t})=t_{1} \mathcal{Y}_{n}\left(0, t_{2}, \ldots\right)$ for $n \geq 0$.

Lemma 2.1 The bivariate exponential generating function for $A_{n+k, k}(\mathbf{t})$ is given by

$$
A(\mathbf{t} ; x, y)=\sum_{n, k \geq 0} A_{n+k, k}(\mathbf{t}) \frac{x^{n}}{n !} \frac{y^{k}}{k !}=t_{1} e^{-x t_{1}} \mathcal{Y}(\mathbf{t} ; x+y)
$$

Proof: Define

$$
A_{k}(\mathbf{t} ; x)=\sum_{n \geq 0} A_{n+k, k}(\mathbf{t}) \frac{x^{n}}{n !} .
$$


Clearly, $A_{0}(\mathbf{t} ; x)=t_{1} e^{-x t_{1}} \mathcal{Y}(\mathbf{t} ; x)$. From (1), one can derive that

$$
A_{k}(\mathbf{t} ; x)=t_{1} A_{k-1}(\mathbf{t} ; x)+\frac{\partial}{\partial x} A_{k-1}(\mathbf{t} ; x),
$$

which produces

$$
A_{k}(\mathbf{t} ; x)=\left(t_{1}+\frac{\partial}{\partial x}\right) A_{k-1}(\mathbf{t} ; x)=\left(t_{1}+\frac{\partial}{\partial x}\right)^{k} A_{0}(\mathbf{t} ; x)
$$

Then

$$
\begin{aligned}
A(\mathbf{t} ; x, y) & =\sum_{k \geq 0} A_{k}(\mathbf{t} ; x) \frac{y^{k}}{k !}=\sum_{k \geq 0} \frac{y^{k}\left(t_{1}+\frac{\partial}{\partial x}\right)^{k}}{k !} A_{0}(\mathbf{t} ; x) \\
& =e^{y t_{1}+y \frac{\partial}{\partial x}} t_{1} e^{-x t_{1}} \mathcal{Y}(\mathbf{t} ; x)=t_{1} e^{y t_{1}} e^{y \frac{\partial}{\partial x}} e^{-x t_{1}} \mathcal{Y}(\mathbf{t} ; x) \\
& =t_{1} e^{y t_{1}} e^{-(x+y) t_{1}} \mathcal{Y}(\mathbf{t} ; x+y)=t_{1} e^{-x t_{1}} \mathcal{Y}(\mathbf{t} ; x+y)
\end{aligned}
$$

This completes the proof.

Theorem 2.2 For any integers $n, m \geq 0$ and any indeterminate $\lambda$, there hold

$$
\begin{aligned}
& \sum_{k=0}^{n}\left(\begin{array}{c}
k+\lambda-1 \\
k
\end{array}\right) A_{n+m, m+k}(\mathbf{t})=\sum_{k=0}^{n}\left(\begin{array}{c}
n+\lambda \\
k
\end{array}\right)\left(\begin{array}{c}
n+\lambda-k-1 \\
n-k
\end{array}\right) A_{m+k, m}(\mathbf{t}) t_{1}^{n-k}, \\
& \sum_{k=0}^{n}\left(\begin{array}{c}
k+\lambda-1 \\
k
\end{array}\right) A_{n+m, m+k}(\mathbf{t})=\sum_{k=0}^{n}(-1)^{n-k}\left(\begin{array}{c}
n+\lambda \\
k
\end{array}\right) \mathcal{Y}_{m+k}(\mathbf{t}) t_{1}^{n-k+1} .
\end{aligned}
$$

Proof: With the umbra $\mathbf{Y}_{\mathbf{t}}$, given by $\mathbf{Y}_{\mathbf{t}}^{n}=\mathcal{Y}_{n}(\mathbf{t}), \mathcal{Y}(\mathbf{t} ; x)$ may be written as $\mathcal{Y}(\mathbf{t} ; x)=e^{\mathbf{Y}_{\mathbf{t}} x}$. (See, for example, [7, 12, 13]). Then, by Lemma 2.1, we have

$$
A(\mathbf{t} ; x, y)=t_{1} e^{\mathbf{Y}_{\mathbf{t}}(x+y)-t_{1} x}=t_{1} e^{\left(\mathbf{Y}_{\mathbf{t}}-t_{1}\right) x} e^{\mathbf{Y}_{\mathbf{t}} y} .
$$

When comparing the coefficient of $\frac{x^{n} y^{k}}{n ! k !}, A_{n+k, k}(\mathbf{t})$ can be represented umbrally as

$$
A_{n+k, k}(\mathbf{t})=t_{1} \mathbf{Y}_{\mathbf{t}}^{k}\left(\mathbf{Y}_{\mathbf{t}}-t_{1}\right)^{n} .
$$

Let $\left[x^{n}\right] f(x)$ denote the coefficient of $x^{n}$ in the formal power series $f(x)$. Then we get

$$
\begin{aligned}
& \sum_{k=0}^{n}\left(\begin{array}{c}
k+\lambda-1 \\
k
\end{array}\right) A_{n+m, m+k}(\mathbf{t}) \\
& \quad=\sum_{k=0}^{n}(-1)^{k}\left(\begin{array}{c}
-\lambda \\
k
\end{array}\right) t_{1} \mathbf{Y}_{\mathbf{t}}^{m+k}\left(\mathbf{Y}_{\mathbf{t}}-t_{1}\right)^{n-k} \\
& \quad=t_{1} \mathbf{Y}_{\mathbf{t}}^{m}\left(\mathbf{Y}_{\mathbf{t}}-t_{1}\right)^{n} \sum_{k=0}^{n}\left(\begin{array}{c}
-\lambda \\
k
\end{array}\right)\left(-\frac{\mathbf{Y}_{\mathbf{t}}}{\mathbf{Y}_{\mathbf{t}}-t_{1}}\right)^{k}
\end{aligned}
$$




$$
\begin{aligned}
& =t_{1} \mathbf{Y}_{\mathbf{t}}^{m}\left(\mathbf{Y}_{\mathbf{t}}-t_{1}\right)^{n} \sum_{k=0}^{n}\left[x^{k}\right]\left(1-\frac{x \mathbf{Y}_{\mathbf{t}}}{\mathbf{Y}_{\mathbf{t}}-t_{1}}\right)^{-\lambda} \\
& =t_{1} \mathbf{Y}_{\mathbf{t}}^{m}\left(\mathbf{Y}_{\mathbf{t}}-t_{1}\right)^{n}\left[x^{n}\right] \frac{1}{1-x}\left(1-\frac{x \mathbf{Y}_{\mathbf{t}}}{\mathbf{Y}_{\mathbf{t}}-t_{1}}\right)^{-\lambda} \\
& =t_{1} \mathbf{Y}_{\mathbf{t}}^{m}\left(\mathbf{Y}_{\mathbf{t}}-t_{1}\right)^{n}\left[x^{n}\right] \frac{1}{(1-x)^{\lambda+1}}\left(1-\frac{x}{(1-x)} \frac{t_{1}}{\left(\mathbf{Y}_{\mathbf{t}}-t_{1}\right)}\right)^{-\lambda} \\
& =t_{1} \mathbf{Y}_{\mathbf{t}}^{m}\left(\mathbf{Y}_{\mathbf{t}}-t_{1}\right)^{n}\left[x^{n}\right] \sum_{k=0}^{n}\left(\begin{array}{c}
-\lambda \\
n-k
\end{array}\right) \frac{x^{n-k}}{(1-x)^{n+\lambda-k+1}}\left(-\frac{t_{1}}{\mathbf{Y}_{\mathbf{t}}-t_{1}}\right)^{n-k} \\
& =\sum_{k=0}^{n}(-1)^{k}\left(\begin{array}{c}
-(n+\lambda-k+1) \\
k
\end{array}\right)\left(\begin{array}{c}
-\lambda \\
n-k
\end{array}\right) t_{1} \mathbf{Y}_{\mathbf{t}}^{m}\left(\mathbf{Y}_{\mathbf{t}}-t_{1}\right)^{k}\left(-t_{1}\right)^{n-k} \\
& =\sum_{k=0}^{n}\left(\begin{array}{c}
n+\lambda \\
k
\end{array}\right)\left(\begin{array}{c}
n+\lambda-k-1 \\
n-k
\end{array}\right) A_{m+k, m}(\mathbf{t}) t_{1}^{n-k},
\end{aligned}
$$

which proves 2 .

By the identity

$$
\left(\begin{array}{l}
n \\
k
\end{array}\right)\left(\begin{array}{l}
k \\
i
\end{array}\right)=\left(\begin{array}{l}
n \\
i
\end{array}\right)\left(\begin{array}{l}
n-i \\
k-i
\end{array}\right),
$$

and Vandermonde's convolution identity

$$
\sum_{k=0}^{n}\left(\begin{array}{l}
a \\
k
\end{array}\right)\left(\begin{array}{c}
b \\
n-k
\end{array}\right)=\left(\begin{array}{c}
a+b \\
n
\end{array}\right),
$$

we have

$$
\begin{aligned}
\sum_{k=0}^{n} & \left(\begin{array}{c}
k+\lambda-1 \\
k
\end{array}\right) A_{n+m, m+k}(\mathbf{t}) \\
& =\sum_{k=0}^{n}\left(\begin{array}{c}
n+\lambda \\
k
\end{array}\right)\left(\begin{array}{c}
-\lambda \\
n-k
\end{array}\right) t_{1} \mathbf{Y}_{\mathbf{t}}^{m}\left(\mathbf{Y}_{\mathbf{t}}-t_{1}\right)^{k}\left(-t_{1}\right)^{n-k} \\
& =\sum_{k=0}^{n}\left(\begin{array}{c}
n+\lambda \\
k
\end{array}\right)\left(\begin{array}{c}
-\lambda \\
n-k
\end{array}\right) \sum_{i=0}^{k}\left(\begin{array}{c}
k \\
i
\end{array}\right) t_{1} \mathbf{Y}_{\mathbf{t}}^{m+i}\left(-t_{1}\right)^{n-i} \\
& =\sum_{i=0}^{n} t_{1} \mathbf{Y}_{\mathbf{t}}^{m+i}\left(-t_{1}\right)^{n-i} \sum_{k=i}^{n}\left(\begin{array}{c}
n+\lambda \\
k
\end{array}\right)\left(\begin{array}{c}
-\lambda \\
n-k
\end{array}\right)\left(\begin{array}{c}
k \\
i
\end{array}\right) \\
& =\sum_{i=0}^{n}\left(\begin{array}{c}
n+\lambda \\
i
\end{array}\right) t_{1} \mathbf{Y}_{\mathbf{t}}^{m+i}\left(-t_{1}\right)^{n-i} \sum_{k=i}^{n}\left(\begin{array}{c}
-\lambda \\
n-k
\end{array}\right)\left(\begin{array}{c}
n+\lambda-i \\
k-i
\end{array}\right) \\
& =\sum_{i=0}^{n}\left(\begin{array}{c}
n+\lambda \\
i
\end{array}\right) t_{1} \mathbf{Y}_{\mathbf{t}}^{m+i}\left(-t_{1}\right)^{n-i}
\end{aligned}
$$




$$
=\sum_{k=0}^{n}(-1)^{n-k}\left(\begin{array}{c}
n+\lambda \\
k
\end{array}\right) \mathcal{Y}_{m+k}(\mathbf{t}) t_{1}^{n-k+1},
$$

which proves 3 .

The case $\lambda=0$ in 3 yields an explicit formula for $A_{n+m, m}(\mathbf{t})$.

Corollary 2.3 For any integers $n, m \geq 0$, there holds

$$
A_{n+m, m}(\mathbf{t})=\sum_{k=0}^{n}(-1)^{n-k}\left(\begin{array}{l}
n \\
k
\end{array}\right) t_{1}^{n-k+1} \mathcal{Y}_{m+k}(\mathbf{t}) .
$$

Corollary 2.4 For any integers $n, m \geq 0$, there hold

$$
\begin{aligned}
\sum_{k=0}^{n} A_{n+m, m+k}(\mathbf{t}) & =\frac{t_{1} \mathcal{Y}_{n+m+1}(\mathbf{t})-A_{n+m+1, m}(\mathbf{t})}{t_{1}} \\
\sum_{k=0}^{n}(k+1) A_{n+m, m+k}(\mathbf{t}) & =\frac{A_{n+m+2, m}(\mathbf{t})-t_{1} \mathcal{Y}_{n+m+2}(\mathbf{t})+(n+2) t_{1}^{2} \mathcal{Y}_{n+m+1}(\mathbf{t})}{t_{1}^{2}} \\
\sum_{k=0}^{n}(n-k+1) A_{n+m, m+k}(\mathbf{t}) & =\frac{t_{1} \mathcal{Y}_{n+m+2}(\mathbf{t})-A_{n+m+2, m}(\mathbf{t})-(n+2) t_{1} A_{n+m+1, m}(\mathbf{t})}{t_{1}^{2}}
\end{aligned}
$$

Proof: By combining (5) with $n$ replaced by $n+1$ (resp. $n+2$ ) and with the case $\lambda=1$ (resp. $\lambda=2$ ) in (3), we obtain (6) (resp. (7)). Moreover, (8) can be easily obtained from (6) and (7).

Theorem 2.5 For any integers $n, m, k \geq 0$, there holds

$$
A_{n+m+k, m+k}(\mathbf{t})=\sum_{j=0}^{m}\left(\begin{array}{c}
m \\
j
\end{array}\right) t_{1}^{m-j} A_{n+k+j, k}(\mathbf{t}) .
$$

Proof: Here we provide a combinatorial proof. For any $\pi \in \mathbb{A}_{n+m+k, m+k}$, suppose that $\pi$ has exactly $m-j$ singletons in $\{k+1, \ldots, k+m\}$, which contributes the weight $t_{1}^{m-j}$, and there are $\left(\begin{array}{c}m \\ j\end{array}\right)$ ways to do this. The remaining $j$ elements in $\{k+1, \ldots, k+m\}$ can not be singletons in $\pi$. These $j$ elements can be regarded as the roles that greater than $m+k+1$, so the remaining $n+k+j+1$ elements can be partitioned with the largest singleton $m+k+1$, these cases contribute the weight $A_{n+k+j, k}(\mathbf{t})$. Thus the total weight of such partitions is $\left(\begin{array}{c}m \\ j\end{array}\right) t_{1}^{m-j} A_{n+k+j, k}(\mathbf{t})$. Summing up all the possible cases yields 9 .

Theorem 2.6 For any integers $n, m \geq 0$ and any indeterminate $y$, there hold

$$
\begin{aligned}
& \sum_{k=0}^{n}\left(\begin{array}{l}
n \\
k
\end{array}\right) A_{n+m, m+k}(\mathbf{t}) y^{k}=\sum_{k=0}^{n}(-1)^{n-k}\left(\begin{array}{l}
n \\
k
\end{array}\right) \mathcal{Y}_{m+k}(\mathbf{t})(y+1)^{k} t_{1}^{n-k+1} \\
& \sum_{k=0}^{n}\left(\begin{array}{l}
n \\
k
\end{array}\right) A_{m+k, m}(\mathbf{t}) y^{n-k}=t_{1} \sum_{k=0}^{n}\left(\begin{array}{l}
n \\
k
\end{array}\right) \mathcal{Y}_{m+k}(\mathbf{t})\left(y-t_{1}\right)^{n-k}
\end{aligned}
$$


Proof: By [4], we have

$$
\begin{aligned}
\sum_{k=0}^{n}\left(\begin{array}{l}
n \\
k
\end{array}\right) A_{n+m, m+k}(\mathbf{t}) y^{k} & =\sum_{k=0}^{n}\left(\begin{array}{l}
n \\
k
\end{array}\right) t_{1} \mathbf{Y}_{\mathbf{t}}^{m+k}\left(\mathbf{Y}_{\mathbf{t}}-t_{1}\right)^{n-k} y^{k} \\
& =t_{1} \mathbf{Y}_{\mathbf{t}}^{m}\left((y+1) \mathbf{Y}_{\mathbf{t}}-t_{1}\right)^{n} \\
& =\sum_{k=0}^{n}(-1)^{n-k}\left(\begin{array}{l}
n \\
k
\end{array}\right)(y+1)^{k} \mathbf{Y}_{\mathbf{t}}^{m+k} t_{1}^{n-k+1} \\
& =\sum_{k=0}^{n}(-1)^{n-k}\left(\begin{array}{l}
n \\
k
\end{array}\right)(y+1)^{k} \mathcal{Y}_{m+k}(\mathbf{t}) t_{1}^{n-k+1},
\end{aligned}
$$

which proves 10. Similarly, 11] can be obtained, but here we provide a combinatorial proof.

Let $\mathbb{X}_{n, m}=\bigcup_{j=0}^{n} \mathbb{X}_{n, m, k}$ and $\mathbb{X}_{n, m, k}$ denote the set of pairs $(\pi, \mathbb{S})$ such that

- $\mathbb{S}$ is an $(n-k)$-subset of $[m+2, n+m+1]=\{m+2, \ldots, n+m+1\}$, and each element of $\mathbb{S}$ is colored by $t_{1}$ or $y-t_{1}$;

- $\pi$ is a partition of the set $[n+m+1]-\mathbb{S}$ with the largest singleton $m+1$, and each element of $[n+m+1]-\mathbb{S}$ is only colored by 1 .

Let $\mathbb{Y}_{n, m}=\bigcup_{k=0}^{n} \mathbb{Y}_{n, m, k}$ and $\mathbb{Y}_{n, m, k}$ denote the set of pairs $(\pi, \mathbb{S})$ such that

- $\mathbb{S}$ is an $(n-k)$-subset of $[m+2, n+m+1]$ and each element of $\mathbb{S}$ is only colored by $y-t_{1}$;

- $\pi$ is a partition of the set $[n+m+1]-\mathbb{S}$ such that $m+1$ must be a singleton, and each element of $[n+m+1]-\mathbb{S}$ is only colored by 1 .

The weight of $(\pi, \mathbb{S})$ is defined to be the product of the weight of $\pi$ and the color of each element of $[n+m+1]$. Clearly, the weights of $\mathbb{X}_{n, m}$ and $\mathbb{Y}_{n, m}$ are counted respectively by the left and right sides of (11).

Given any pair $(\pi, \mathbb{S}) \in \mathbb{X}_{n, m}, \mathbb{S}$ can be partitioned into two parts $\mathbb{S}_{1}$ and $\mathbb{S}_{2}$ such that each element of $\mathbb{S}_{1}$ is colored by $y-t_{1}$ and each element of $\mathbb{S}_{2}$ is colored by $t_{1}$. Regard each element of $\mathbb{S}_{2}$ as a singleton which is weighted by $t_{1}$ and colored by 1 , together with $\pi$, we obtain a partition $\pi_{1}$ of $[n+m+1]-\mathbb{S}_{1}$ such that $m+1$ is always a singleton. Then the pair $\left(\pi_{1}, \mathbb{S}_{1}\right)$ lies in $\mathbb{Y}_{n, m}$.

Conversely, for any pair $\left(\pi_{1}, \mathbb{S}_{1}\right) \in \mathbb{Y}_{n, m}$, let $\mathbb{S}$ denote the union of $\mathbb{S}_{1}$ and the singletons of $\pi_{1}$ greater than $m+1$, then $\pi_{1}$ can be partitioned into two parts $\pi$ and $\pi^{\prime}$ such that $\pi$ is a partition of $[n+m+1]-\mathbb{S}$ with the largest singleton $m+1$ and $\pi^{\prime}$ is the singletons of $\pi_{1}$ greater than $m+1$. By regarding $\pi^{\prime}$ as a subset of $[m+2, n+m+1]$ in which each element is colored by $t_{1}$, together with $\mathbb{S}_{1}$. Then we obtain an $(n-k)$-subset of $[m+2, n+m+1]$ for some $k$ such that each element of $\mathbb{S}$ is colored by $t_{1}$ or $y-t_{1}$. Then the pair $(\pi, \mathbb{S})$ lies in $\mathbb{X}_{n, m}$.

Clearly we find a bijection between $\mathbb{X}_{n, m}$ and $\mathbb{Y}_{n, m}$, which proves (11).

The cases $y=-1$ in (10) and $y=t_{1}$ in (11) lead to 
Corollary 2.7 For any integers $n, m \geq 0$, there hold

$$
\begin{gathered}
\sum_{k=0}^{n}(-1)^{n-k}\left(\begin{array}{l}
n \\
k
\end{array}\right) A_{n+m, m+k}(\mathbf{t})=\mathcal{Y}_{m}(\mathbf{t}) t_{1}^{n+1}, \\
\sum_{k=0}^{n}\left(\begin{array}{l}
n \\
k
\end{array}\right) A_{m+k, m}(\mathbf{t}) t_{1}^{n-k-1}=\mathcal{Y}_{m+n}(\mathbf{t}) .
\end{gathered}
$$

The case $y=\frac{y t_{1}}{y+1}$ in 11, , together with 10 generates the following result which has a combinatorial interpretation.

Corollary 2.8 For any integers $n, m \geq 0$, there holds

$$
\sum_{k=0}^{n}\left(\begin{array}{l}
n \\
k
\end{array}\right) A_{m+k, m}(\mathbf{t})(y+1)^{k}\left(y t_{1}\right)^{n-k}=\sum_{k=0}^{n}\left(\begin{array}{l}
n \\
k
\end{array}\right) A_{n+m, m+k}(\mathbf{t}) y^{k} .
$$

Proof: Let $\mathbb{X}_{n, m}^{*}=\bigcup_{j=0}^{n} \mathbb{X}_{n, m, k}^{*}$ and $\mathbb{X}_{n, m, k}^{*}$ denote the set of pairs $(\pi, \mathbb{S})$ such that

- $\pi$ is a partition of the set $[n+m+1]$ containing at least the singleton $m+1$;

- $\mathbb{S}$ is an $(n-k)$-subset of $[m+2, n+m+1]$ which is also the set of singletons of $\pi$ greater than $m+1$, each element of $\mathbb{S}$ is only colored by $y$ and each element of $[m+2, n+m+1]-\mathbb{S}$ is colored by 1 or $y$;

- each element of $[m+1]$ is only colored by 1 .

Let $\mathbb{Y}_{n, m}^{*}=\bigcup_{k=0}^{n} \mathbb{Y}_{n, m, k}^{*}$ and $\mathbb{Y}_{n, m, k}^{*}$ denote the set of pairs $(\pi, \mathbb{S})$ such that

- $\mathbb{S}$ is a $k$-subset $\left\{i_{1}, i_{2}, \ldots, i_{k}\right\}$ of $[m+2, n+m+1]$ in increasing order, each element of $\mathbb{S}$ is only colored by $y$ and each element of $[n+m+1]-\mathbb{S}$ is only colored by 1 ;

- $\pi$ is a partition of the set $[n+m+1]$ such that $i_{k}$ must be the largest singleton if $\mathbb{S}$ is not empty and $m+1$ must be the largest singleton if $\mathbb{S}$ is empty;

- each element of $[m+2, n+m+1]-\mathbb{S}$ must not be a singleton.

The weight of $(\pi, \mathbb{S})$ is defined to be the product of the weight of $\pi$ and the colors of all elements in $[n+m+1]$. Clearly, any $(\pi, \mathbb{S}) \in \mathbb{X}_{n, m}^{*}$ can be obtained as follows. First choose an $(n-k)$-subset $\mathbb{S}$ of $[m+2, n+m+1]$, there are $\left(\begin{array}{l}n \\ k\end{array}\right)$ ways to do this. Regard each element of $\mathbb{S}$ as a singleton with color $y$. Then color each element of $[m+2, n+m+1]-\mathbb{S}$ by 1 or $y$, namely, each element of $[m+2, n+m+1]-\mathbb{S}$ is colored by $y+1$. Now partitioning $[n+m+1]-\mathbb{S}$ such that the largest singleton is $m+1$, together with the $n-k$ singletons formed form $\mathbb{S}$, we get the partition $\pi$ of $[n+m+1]$ such that $m+1$ must be a singleton; Hence the total weight of pairs $(\pi, \mathbb{S}) \in \mathbb{X}_{n, m}^{*}$ is just the left hand side of $(12)$.

Similarly, the total weight of pairs $(\pi, \mathbb{S}) \in \mathbb{Y}_{n, m}^{*}$ is just the right hand side of $(12)$ if regarding each element of $[m+2, n+m+1]-\mathbb{S}$ as the role greater than $i_{k}$ when $\mathbb{S} \neq \emptyset$.

Now we can construct a bijection $\varphi$ between $\mathbb{X}_{n, m}^{*}$ and $\mathbb{Y}_{n, m}^{*}$ which preserves the weights. For any $(\pi, \mathbb{S}) \in \mathbb{X}_{n, m}^{*}$, let $\mathbb{S}_{1}$ denote the set of elements of $[n+m+1]$ with colors $y$. Clearly, $\mathbb{S}$ is a subset of $\mathbb{S}_{1}$. 
Assume that $\mathbb{S}_{1}=\left\{i_{1}, i_{2}, \ldots, i_{k}\right\}$ for some $0 \leq k \leq n$ in increasing order. If $\mathbb{S}_{1}$ is the empty set $\emptyset$, which implies that $\mathbb{S}=\emptyset$ and all elements of $[n+m+1]$ are colored by 1 , it is obvious that $(\pi, \emptyset) \in \mathbb{Y}_{n, m}^{*}$. Then define $\varphi(\pi, \emptyset)=(\pi, \emptyset)$. If $\mathbb{S}_{1}$ is not the empty set, exchanging $m+1$ and $i_{k}$ in $\pi$, we obtain a partition $\pi_{1}$, it is easily to verify that $\left(\pi_{1}, \mathbb{S}_{1}\right) \in \mathbb{Y}_{n, m}^{*}$ and has the same weight as $(\pi, \mathbb{S})$. Then define $\varphi(\pi, \mathbb{S})=\left(\pi_{1}, \mathbb{S}_{1}\right)$

Conversely, for any $\left(\pi_{1}, \mathbb{S}_{1}\right) \in \mathbb{Y}_{n, m}^{*}$, if $\mathbb{S}_{1}=\emptyset$, so $\pi_{1}$ has the largest singleton $m+1$, then $\left(\pi_{1}, \emptyset\right) \in$ $\mathbb{X}_{n, m}^{*}$ and define $\varphi^{-1}\left(\pi_{1}, \emptyset\right)=\left(\pi_{1}, \emptyset\right)$. If $\mathbb{S}_{1} \neq \emptyset$, assume that $\mathbb{S}_{1}=\left\{i_{1}, i_{2}, \ldots, i_{k}\right\}$ for some $1 \leq k \leq n$ in increasing order, let $\mathbb{S}$ denote the set of all the elements in $\mathbb{S}_{1}$ such that each forms a singleton of $\pi_{1}$. Now exchanging $m+1$ and $i_{k}$ in $\pi_{1}$, we obtain a partition $\pi$, it is easy verifiable that $(\pi, \mathbb{S}) \in \mathbb{X}_{n, m}^{*}$ which has the same weight as $\left(\pi_{1}, \mathbb{S}_{1}\right)$. Then define $\varphi^{-1}\left(\pi_{1}, \mathbb{S}_{1}\right)=(\pi, \mathbb{S})$.

Clearly, $\varphi$ is indeed a bijection between $\mathbb{X}_{n, m}^{*}$ and $\mathbb{Y}_{n, m}^{*}$, which proves 12 .

\section{The special case for permutations}

When the parameter $\mathbf{t}$ in $A_{n, k}(\mathbf{t})$ takes some special value, that is to assign a special structure to each block of partitions of $[n+1]$. For example, the case $\mathbf{t}=\left(1^{0}, 2^{1}, 3^{2}, \ldots\right)$ indicates that each block of partitions is assigned by a (rooted and labeled) tree structure, such partitions are equivalent to labeled forests; The case $\mathbf{t}=(1,1,0, \ldots)$ leads to involutions on $[n+1]$.

In this section, we just present an interesting specialization, but leave others to inclined readers. Consider the special case when $\mathbf{t}=(0 !, 1 !, 2 !, \ldots)$, that is to assign a cycle structure to each block of partitions, such partitions is equivalent to permutations. Let $P_{n, k}=A_{n, k}(\mathbf{t})$ with $\mathbf{t}=(0 !, 1 !, 2 !, \ldots)$, i.e., $P_{n, k}$ is the number of permutations of $[n+1]$ with the largest fixed point $k+1$. From $[5$ and $(9)$, one has the explicit formulas for $P_{n, k}$

$$
P_{n+k, k}=\sum_{j=0}^{n}(-1)^{n-j}\left(\begin{array}{l}
n \\
j
\end{array}\right)(k+j) !=\sum_{j=0}^{k}\left(\begin{array}{l}
k \\
j
\end{array}\right) D_{n+j} .
$$

Clearly, $P_{n, n}=n !=\mathcal{Y}_{n}(0 !, 1 !, 2 !, \ldots)$ and $P_{n, 0}=D_{n}=\mathcal{Y}_{n}(0,1 !, 2 !, \ldots)$, where $D_{n}$ is the derangement number of $[n]$, i.e., the number of permutations of $[n]$ without fixed points. See Table 1 for some small values of $P_{n, k}$.

\begin{tabular}{c|ccccccc}
\hline$n / k$ & 0 & 1 & 2 & 3 & 4 & 5 & 6 \\
\hline 0 & 1 & & & & & & \\
1 & 0 & 1 & & & & & \\
2 & 1 & 1 & 2 & & & & \\
3 & 2 & 3 & 4 & 6 & & & \\
4 & 9 & 11 & 14 & 18 & 24 & & \\
5 & 44 & 53 & 64 & 78 & 96 & 120 & \\
6 & 265 & 309 & 362 & 426 & 504 & 600 & 720 \\
\hline
\end{tabular}

Table 1. The values of $P_{n, k}$ for $n$ and $k$ up to 6 . 
In fact $\left\{P_{n, k}\right\}_{n \geq k \geq 0}$ forms the difference table introduced by Euler, which has been investigated in depth in derangement theory [2, 5, 6, 8, 9]. Chen [1] gave two other interpretations for $P_{n, k}$ using $k$ relative derangements on $[n]$ and skew derangements from $[n]$ to $\{-k+1, \ldots,-1,0,1, \ldots, n-k\}$ for $0 \leq k \leq n$. Moreover, Chen established a bijection between these two settings. Recently, Deutsch and Elizalde [4] gave a new interpretation of derangement number $D_{n+2}$ as the sum of the values of the largest fixed points of all non-derangements of length $n+1$, namely,

$$
\sum_{k=0}^{n}(k+1) P_{n, k}=D_{n+2}
$$

which is the special case of $(7)$ when $\mathbf{t}=(0 !, 1 !, 2 !, \ldots)$ and $m=0$.

Next, we can explore some new relations between $P_{n, k}$ and other classical sequences such as Bell numbers or Fibonacci numbers.

Example 3.1 By Lemma 2.1, one can derive the bivariate exponential generating function for $P_{n+k, k}$, i.e.,

$$
P(x, y)=\sum_{n, k \geq 0} P_{n+k, k} \frac{x^{n}}{n !} \frac{y^{k}}{k !}=\frac{e^{-x}}{1-x-y}
$$

Extracting the coefficient of $\frac{x^{n}}{n !}$ in $P\left(x, x^{2}\right)$, we have

$$
\sum_{k=0}^{[n / 2]}\left(\begin{array}{c}
n \\
2 k
\end{array}\right)\left(\begin{array}{c}
2 k \\
k
\end{array}\right) k ! P_{n-k, k}=\sum_{k=0}^{n}(-1)^{n-k}\left(\begin{array}{l}
n \\
k
\end{array}\right) k ! F_{k}
$$

where $F_{k}$ is the $k$-th Fibonacci number defined by $\frac{1}{1-x-x^{2}}=\sum_{k \geq 0} F_{k} x^{k}$.

Example 3.2 When $\mathbf{t}=(0 !, 1 !, 2 !, \ldots),(10)$ and $(11)$ reduce to

$$
\begin{aligned}
& \sum_{k=0}^{n}\left(\begin{array}{l}
n \\
k
\end{array}\right) P_{n+m, m+k} y^{k}=\sum_{k=0}^{n}(-1)^{n-k}\left(\begin{array}{l}
n \\
k
\end{array}\right)(m+k) !(y+1)^{k}, \\
& \sum_{k=0}^{n}\left(\begin{array}{l}
n \\
k
\end{array}\right) P_{m+k, m} y^{n-k}=\sum_{k=0}^{n}\left(\begin{array}{l}
n \\
k
\end{array}\right)(m+k) !(y-1)^{n-k} .
\end{aligned}
$$

It should be noted that (13) and (14) have close relations to the (re-normalized) Charlier polynomials $C_{n}(u, v)$ [7] defined by

$$
C_{n}(u, v)=\sum_{k=0}^{n}\left(\begin{array}{l}
n \\
k
\end{array}\right)(u)_{k} v^{n-k}
$$

where $(u)_{k}=u(u+1) \cdots(u+k-1)$ for $k \geq 1$ and $(u)_{0}=1$. In fact 13$)$ is $\frac{(y+1)^{n}}{m !} C_{n}\left(m+1,-\frac{1}{y+1}\right)$ and 14 is equal to $\frac{1}{m !} C_{n}(m+1, y-1)$. 
Recall that, by 44, $P_{n, k}$ can be represented umbrally as

$$
P_{n, k}=\mathbf{P}^{k}(\mathbf{P}-1)^{n-k},
$$

where $\mathbf{P}=\mathbf{Y}_{\mathbf{t}}$ with $\mathbf{t}=(0 !, 1 !, 2 !, \ldots)$. In particular, $D_{n}=(\mathbf{P}-1)^{n}$ and $n !=\mathbf{P}^{n}$. Hence, the case $y=\mathbf{P}-1$ in $(13)$ and the case $y=\mathbf{P}$ in 14 ) generate

$$
\begin{gathered}
\sum_{k=0}^{n}\left(\begin{array}{l}
n \\
k
\end{array}\right) P_{n+m, m+k} D_{k}=\sum_{k=0}^{n}(-1)^{n-k}\left(\begin{array}{l}
n \\
k
\end{array}\right)(m+k) ! k !, \\
\sum_{k=0}^{n}\left(\begin{array}{l}
n \\
k
\end{array}\right) P_{m+k, m}(n-k) !
\end{gathered}
$$

With the Bell umbra $\mathbf{B}[\mathbf{7}, 12,13]$, given by $\mathbf{B}=\mathbf{Y}_{\mathbf{t}}$ with $\mathbf{t}=(1,1,1, \ldots)$, the Bell number can be written as $B_{n}=\mathbf{B}^{n}$ and $\mathbf{B}^{n+1}=(\mathbf{B}+1)^{n}$. Then the case $y=\mathbf{B}$ in 13 and the case $y=\mathbf{B}+1$ in 14 generate

$$
\begin{aligned}
\sum_{k=0}^{n}\left(\begin{array}{l}
n \\
k
\end{array}\right) P_{n+m, m+k} B_{k} & =\sum_{k=0}^{n}(-1)^{n-k}\left(\begin{array}{l}
n \\
k
\end{array}\right)(m+k) ! B_{k+1}, \\
\sum_{k=0}^{n}\left(\begin{array}{l}
n \\
k
\end{array}\right) P_{m+k, m} B_{n-k+1} & =\sum_{k=0}^{n}\left(\begin{array}{l}
n \\
k
\end{array}\right)(m+k) ! B_{n-k} .
\end{aligned}
$$

Using the Riordan identity [3, 11, P173],

$$
\sum_{k=0}^{n}\left(\begin{array}{l}
n \\
k
\end{array}\right)(k+1) !(n+1)^{n-k}=(n+1)^{n+1},
$$

the case in 13 with $m=1$ and $y=-\frac{n+2}{n+1}$ and the case in 14 with $m=1$ and $y=n+2$ generate respectively

$$
\begin{aligned}
\sum_{k=0}^{n}(-1)^{n-k}\left(\begin{array}{l}
n \\
k
\end{array}\right) P_{n+1, k+1}(n+2)^{k}(n+1)^{n-k} & =(n+1)^{n+1}, \\
\sum_{k=0}^{n}\left(\begin{array}{l}
n \\
k
\end{array}\right)\left(D_{k}+D_{k+1}\right)(n+2)^{n-k} & =(n+1)^{n+1},
\end{aligned}
$$

where we use the relation $P_{k+1,1}=D_{k}+D_{k+1}$. By the well-known recurrence $D_{k+2}=(k+1)\left(D_{k}+\right.$ $D_{k+1}$ ) for derangement numbers $D_{k}$, together with $D_{1}=0$, after routine computation, 15 is equivalent to

$$
\sum_{k=0}^{n}\left(\begin{array}{l}
n \\
k
\end{array}\right) D_{k+1}(n+1)^{n-k}=n^{n+1},
$$

which was also obtained by Riordan [10]. In a forthcoming paper [18], using functional digraph theory, we will give a combinatorial interpretation for a more general identity involving the Riordan identity and (16) as special cases. 
The largest singletons in weighted set partitions and its applications

\section{Acknowledgements}

The authors are grateful to the anonymous referees for the helpful suggestions and comments.

\section{References}

[1] W. Y. C. Chen, The skew, relative, and classical derangements, Discrete Mathematics 160 (1996), 235-239.

[2] R. J. Clarke, G.-N. Han, and J. Zeng, A combinatorial interpretation of the Seidel generation of q-derangement numbers, Annals of Combinatorics 4 (1997), 313-327.

[3] L. Comtet, Advanced Combinatorics, D. Reidel Publishing Company, Dordrecht-Holland, 1974.

[4] E. Deutsch and S. Elizalde, The largest and the smallest fixed points of permutations, Europ. J. Combin., 31 (5) (2010), 1404-1409.

[5] D. Dumont, Matrices d'Euler-Seidel, Séminaire Lotharingien de Combinatoire, B05c (1981), http:// cartan.u-strasbg.fr $/ \sim$ slc.

[6] D. Dumont and A. Randrianarivony, Dérangements et nombres de Genocchi, Disc. Math., 132 (1994), 37-49.

[7] I. M. Gessel, Applications of the classical umbral calculus, Algebra Universalis, 49 (2003), 397-434.

[8] F. Rakotondrajao, k-Fixed-points permutations, Integers: Electr. J. Combin. Numb. Theo. 7 (2007), \#A36.

[9] F. Rakotondrajao, On Euler's difference table, Proc. FPSAC'07, Nankai University, Tianjin, China (2007).

[10] J. Riordan, Enumeration of linear graphs for mappings of finite sets, Ann. Math. Statist. 33 (1962), 178-185.

[11] J. Riordan, Forests of labeled trees, J. Combinatorial Theory 5 (1968), 90-103.

[12] S. Roman, The Umbral Calculus, Academic Press, Orlando, FL, 1984.

[13] S. Roman and G.-C. Rota, The umbral calculus, Adv. Math. 27 (1978), 95-188.

[14] G. C. Rota, The number of partitions of a set, Amer. Math. Monthly 71 (1964), 498-504.

[15] N.J.A. Sloane, The On-Line Encyclopedia of Integer Sequences, http://www.research.att.com/ njas/sequences.

[16] R. Stanley, Enumerative Combinatorics, vol. 1, Cambridge Univ. Press, Cambridge, 1997.

[17] Y. Sun and X. Wu, The largest singletons of set partitions, Europ. J. Combinatorics, 32 (2011), 369-382.

[18] Y. Sun and J. Zhuang, $\lambda$-factorials of n, Electr. J. Combinatorics 17(1) (2010), \#169. 
Yidong Sun and Yanjie Xu 